\title{
TESTING OF IP SERVICES \& APPLICATIONS IN A SERVICE PROVIDER ENVIRONMENT
}

\author{
Andreas Gehring
}

T-Systems Nova, Technologiezentrum, Germany

\begin{abstract}
A service providers (SP) environment requires several stages and types of testing and quality assurance (QA) before a new service or application can be launched in the network environment. The test procedures cover the whole range from evaluation of single network and server platform components to end-2-end testing depending on the type of application or product. This includes as well technical parameters like QoS in the network and availability of the service as user oriented parameters like usability and user friendliness. This keynote introduces categories of services \& applications and gives an overview of the principle testing methods for the single service categories. It describes the stages of a product roll-out of a SP and the tests which are applied to the service components in each phase.
\end{abstract}

The original version of this chapter was revised: The copyright line was incorrect. This has been corrected. The Erratum to this chapter is available at DOI: 10.1007/978-0-387-35497-2_31 